study was to evaluate the performance of the cobas ${ }^{\boxplus S V} 1$ and 2 test in a Japanese population.

Methods A total of 165 specimens of anogential lesions and endocervcal swabs from 122 patients were tested from clinical sites in Tokyo, Kanagawa and Osaka (36\% Female, 64\% Male). Specimens were tested by cobas HSV 1 and 2 test (cHSV) and compared to shell vial culture (SV) as well as Prime Check HSV Antigen Detection Kit (PCA) and direct immunofluorescence (DFA). Diagnostic agreement was evaluated and discrepant analysis was performed by Sanger sequencing.

Results The overall percent agreement (OPA), sensitivity and specificity of cHSV compared to SV for HSV-1 was $98.8 \%$ (95\% CI 95.7-99.9), 100\% (95\% CI 97.8\%-100\%), and 98.5\% (95\% CI 95.3-99.8), and for HSV-2 was $87.9 \%(95 \%$ CI $81.9-92.5), 98.6 \%$ (95\% CI 95.4\%-99.8\%), and $79.6 \%$ (95\% CI 72.6\%-85.4\%), respectively. For HSV-1 there were 2 cHSV+/SV-. For HSV-2 there were 20 discordant results, 19 $\mathrm{cHSV}+/ \mathrm{SV}$ - and $1 \mathrm{cHSV}-/ \mathrm{SV}+$. Sanger Sequencing for HSV-1 confirmed 1 of 2 positive for HSV-1 and for HSV-2 confirmed 20/20 as HSV-2 positive. Both PCA and DFA missed more than twice as many confirmed positive HSV specimens as SV. The OPA, sensitivity and specificity of cHSV compared to DFA for HSV-1 was $84.8 \%$ (95\% CI 78.4-89.9), $85.7 \%$ (95\% CI 79.4\%-90.7\%), and 84.7\% (95\% CI 78.3\%-89.8\%), and for HSV-2 was $59.8 \%$ (95\% CI 51.9\%-67.4\%), $100 \%$ (95\% CI 97.8\%-100\%), and 53.2\% (95\% CI 45.3\%-61.0\%), respectively. PCA does not type positive HSV. The OPA, sensitivity and specificity of cHSV compared to PCA was $74.5 \%$ (95\% CI 67.1\%-81.0\%), 98.8\% (95\% CI 95.6\%-99.8\%), and $51.8 \%$ (95\% CI 43.9\%-59.6\%).

Conclusion This study demonstrated improved diagnostic performance of the cobas HSV 1 and 2 test compared to routine methods in a Japanese population.

\section{P1.19 HIGH-THROUGHPUT IDENTIFICATION OF SEXUALLY TRANSMITTED INFECTIONS AND BACTERIAL VAGINOSIS ASSOCIATED PATHOGENS ON OPENARRAYTM NANOFLUIDICS QPCR PLATFORM IN SOUTH AFRICA}

${ }^{1}$ Emily Norman, ${ }^{2}$ Dominique Dewulf, ${ }^{3}$ Venessa Maseko, ${ }^{4}$ Joanne Bradfield, ${ }^{5}$ Sunali Patel, ${ }^{5}$ Nivashnee Naicker, ${ }^{5}$ Natasha Samsunder, ${ }^{4}$ Peter Jacobs, ${ }^{5}$ Nigel Garrett. ${ }^{1}$ Columbia University, New York, USA; ${ }^{2}$ Thermofisher Scientific, Massachusetts, USA, Belgium; ${ }^{3}$ Centre for The AIDS Programme of Research in South Africa, Durban, South African Republic; ${ }^{4}$ Thermofisher Scientific, Massachusetts, USA; ${ }^{5}$ Centre for The AIDS Programme of Research in South Africa, Durban, South African Republic

10.1136/sextrans-2017-053264.127

Introduction Cheap and efficient pathogen detection solutions are required to replace syndromic STI management in low and middle income countries. One solution may be point-ofcare technologies at clinic level, another could be centralised high-throughput technologies. ThermoFisher recently launched the TaqMan Vaginal Microbiota Assays, in combination with OpenArray Nanofluidics qPCR platform, which is capable of testing 192 samples for 34 individual STI and bacterial vaginosis (BV) pathogens in a 2 hour qPCR run. The goal of this study was to evaluate OpenArray against an established multiplex PCR assay, and further optimise its workflow.

Methods Evaluation of the TaqMan Vaginal Microbiota assays on OpenArray platform was performed for 50 vaginal micobiota vaginal swab samples that had been characterised for $N$. gonorrheae (NG), C. trachomatis (CT), and T. vaginalis (TV) on an established CDC-approved multiplex PCR assay. Blind samples were provided for testing on the OpenArray platform. Nugent scores were obtained in parallel to molecular testing and results were compared for 11 specific bacterial strains indicative of BV.

Results High specificity (97.4\%-100\%) was observed at initial testing of STI samples, however the sensitivity was not as expected (NG $81.8 \%$, CT $38.5 \%$, TV 50.0\%) due to concentrations of STI pathogens below the limit of detection on OpenArray, which was confirmed by 384 -well plate testing ( $\mathrm{C}_{\mathrm{RT}}$ range 33-38). Pre-amplification of STI samples improved the sensitivity significantly (NG 100\%, CT 92\%, TV 82\%). Nugent scores for 46/50 samples were compared with the qPCR results for the BV-associated targets on OpenArray. BVassociated pathogens like $G$. vaginalis, A. vaginae, BVAB2, Megasphaera 1, Megasphara 2, M.hominis, and M. mulieris were predominate in the samples with Nugent Scores 7-10, while commensal lactobacillus were predominate in Nugent Scores 0-3.

Conclusions After optimisation, the OpenArray Nanofluidics qPCR platform may provide a high-throughput solution for STI pathogen detection and for characterising the vaginal microbiota.

\section{P1.20 ANALYSIS OF BACTERIAL DIVERSITY IN HIV/HPV COINFECTED PATIENTS WITH CERVICAL INTRAEPITHELIAL LESIONS THROUGH NEXT- GENERATION SEQUENCING}

${ }^{1}$ Gislaine Curty Ferreira, ${ }^{2}$ Costa Rl, ${ }^{2}$ Siqueira Jd, ${ }^{3}$ Meyrelles Ai, ${ }^{3}$ Machado Es, ${ }^{2}$ Soares Ea, ${ }^{2}$ Soares Am. 'Instituto Nacional do Câncer, Rio de Janeiro - RJ, Brazil; ${ }^{2}$ Instituto Nacional de Câncer, Rio de Janeiro - RJ, Brazil; ${ }^{3}$ Universidade Federal do Rio De Janeiro, Rio de Janeiro - RJ, Brazil

\subsection{6/sextrans-2017-053264.128}

Introduction Several studies have shown an increase in bacterial diversity in HPV-positive cervical cancer patients and the association of specific bacteria with cervical intraepithelial lesions. However, little is known about the cervical microbiome of HIV/HPV coinfected patients. HIV patients have a high prevalence of high-risk HPV and a greater chance of developing persistent HPV infection. The aim of this study is to evaluate the bacterial profiles of the cervical region of HIV/HPV coinfected patients, looking for a putative association of such profiles with cervical intraepithelial lesions.

Methods We analysed $89 \mathrm{HIV}^{+}$cervical smear samples of women collected from 2010 to 2013. Samples have been categorised according to collection timepoint, $\mathrm{CD}^{+}{ }^{+}$T-cell counts and cervical intraepithelial lesions (CIN). The bacterial 16S rRNA gene was PCR-amplified and processed for next-generation sequencing in an Illumina HiSeq 2500 platform. After sequencing, reads were processed and compared against the 16S database. All bioinformatics analyses were carried out using QIIME.

Results The most abundant bacterial species found was Lactobacillus iners. We found a negative association of the Moryella genus with CIN, independent of the collection timepoint. On the other hand, we observed increased abundance of Gardnerella vaginalis, Shuttleworthia, Veillonellaceae and Aerococcus in CIN, but adjusted p-values were non-significant after false discovery rate and/or Bonferroni corrections.

Conclusion This is first study reporting the Moryella genus in $\mathrm{HIV} / \mathrm{HPV}$ coinfected women and its potential absence in CIN. 